\title{
The Red Queen process does not select for high recombination rates in haplodiploid hosts
}

\author{
J. Kidner \& R.A.F. Moritz
}

J. Kidner

Fachbereich Biology/Martin-Luther-University, Insitute of Biology, Halle/S., Sachsen-Anhalt, Germany

Tel.: +49-(0)345-5526219

Fax: +49-(0)345-5527264

E-mail: jonathan.kidner@zoologie.uni-halle.de

R. A. Moritz

Fachbereich Biology/Martin-Luther-University, Insitute of Biology, Halle/S., Sachsen-Anhalt, Germany

Department of Zoology and Entomology/University of Pretoria, Social Insect Res Grp, Pretoria, 0002, South Africa

\begin{abstract}
One of the main competing theories to describe the evolution of recombination is the Red Queen Hypothesis $(\mathrm{RQH})$. Presently, many theoretical analyses of the RQH typically examine fitness interactions in host-parasite frameworks. Less emphasis has been placed on understanding the impact of host ploidy in these systems. In this study, we look to investigate the high observed rates of recombination observed in two common haplodiploid species (Apis mellifera and Bombus terrestris). We compared haplodiploid to diploid host populations under infection with haploid asexual parasites, using a Matching Allele (MAM) model. Results from a simulation analysis showed that the Red Queen does not run in haplodiploid hosts and is therefore, probably not responsible for the high recombination rates observed so far in haplodiploid hosts.
\end{abstract}

Keywords Red Queen Hypothesis, Hymenoptera, male-haploid, haplodiploid, host-parasite 


\section{Introduction}

Sex is a costly process and the evolution of sexual recombination is an old puzzle of evolutionary biology (Charlesworth 1980). Nevertheless, there are purported advantages to sex as posited by the Red Queen Hypothesis (RQH) (Hamilton 1964). The hypothesis predicts that resistance in host populations infected by multiple parasite strains is maintained through selection for both sex and recombination. The hypothesis requires the presence of negative feedback loops (over generations), whereby common parasite strains select for specific resistance alleles within host populations, which subsequently reduce the prevalence of these strains. This process is similar to negative frequency dependent selection, maintaining genetic variance in both host and parasite populations over generations (Sutton et al. 2011; Takahashi et al. 2011). Sexual recombination may prove beneficial to a host because a genetic association with the most abundant parasite strain would be reduced in subsequent generations. A further potential benefit of sex in the RQH is through the increased amount of genetic variation generated by the reassociation of genetic elements (Barton 1995; Peters and Lively 2007).

Currently, predictions arising from the RQH have been tested using either diploid (SchmidHempel and Jokela 2002; Agrawal and Otto 2006; Agrawal 2009), or haploid (Peters and Lively 2007; Salathé et al 2008; Otto and Nuismer 2004; Peters and Lively 1999) host genetic models. In further studies, haploidy was found to be beneficial for parasites (Nuismer and Otto 2004), whereas higher ploidy levels (diploidy, tetraploidy) were favoured in the host (M'Gonigle and Otto 2011; Oswald and Nuismer 2007). The only exception was the evolution of haploid hosts under the MAM (Nuismer and Otto 2004). The majority of these studies have focused on three interaction models: the Matching Allele Model (MAM), Inverse MAM (IMAM), and the Gene for Gene (GfG); each of which has its own applicability to certain host-parasite systems. The GfG model was, for example, developed from immunology studies on plants (typically rusts; Keen 1990). Likewise the IMAM was developed on the basis of host recognition and response to parasite infection (an adaptive immune response), while the MAM is based on parasite recognition of the host. Since arthropods are not very well known for adaptive immune systems the MAM was adopted as the interaction model over the IMA and GfG models (Wilfert, Gadau and Schmid-Hempel 2007a). However, since the results of previous studies (Nuismer and Otto 2004; Oswald and Nuismer 2007; M'Gonigle and Otto 2011) suggest the possibility of interdependence between these factors, the results here remain specific to the MAM.

We followed a model system close to that of those arthropods that have been shown to exhibit the highest known recombination rates in Bilateria (social insects, Wilfert et al. 2007b; Bumblebees, Stolle et al. 2011; and Honeybees, Meznar et al. 2010; Solignac et al. 2004). All of 
which occur in the order Hymenoptera, an order well known for two features: sociality and haplodiploidy (Normark 2003). While most of those species whose genomes have been well studied were either primitively-, or eu-social, sociality is a derived, polyphyletic trait within the Hymenoptera whereas haplodiploidy is an ancestral, monophyletic trait common to all Hymenoptera. One of the main theories on the origin of haplodiploidy (allelic penetrance: stronger phenotypic representation of alleles) may have very strong relevance in regards to the RQH (Smith 2000). We therefore incorporate the haplodiploid sex determination system in the models presented here. We do not address sociality at this stage though sociality may remain a factor interfering with the evolution of high recombination rates in the social Hymenoptera (differences in recombination rate have been observed between parasitic wasps and eusocial species; Niehuis et al. 2010).

The model

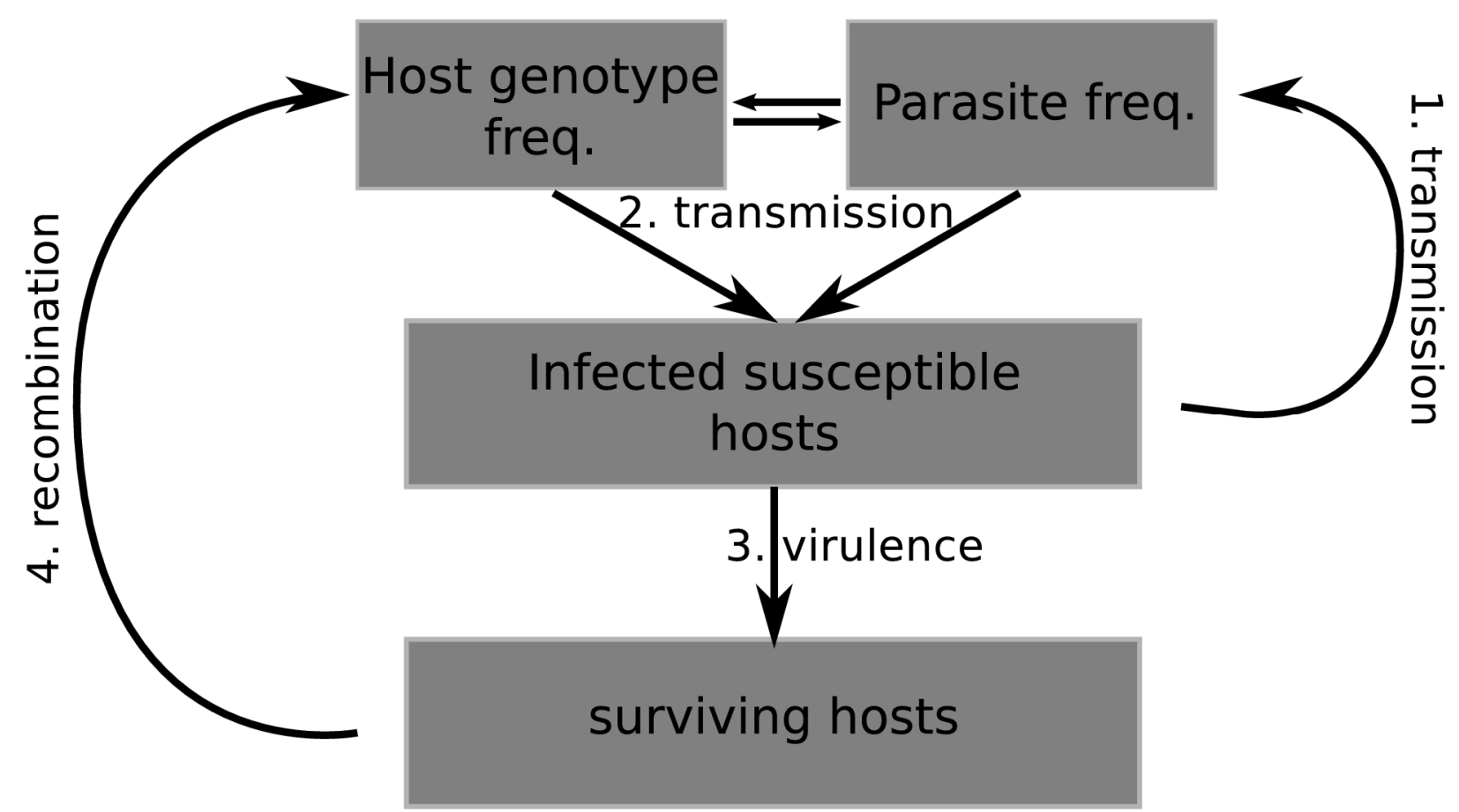

Figure 1: A schematic overview of the model including either a diploid or a haplodiploid host, with a haploid parasite. The model is based on Hardy-Weinberg assumptions with a deterministic population genetic approach under a two loci, two allele model (noted A/a and $B / b)$. The first transmission step is used to indicate inter-generational transmission, while the second is for intra-generational transmission. 
We created both diploid and haplodiploid host models, with a haploid parasite under HardyWeinberg assumptions. It is based on a simple deterministic population genetic approach with a two loci model (noted $A$ and $B$ fig 1), with two alleles each (indicated by the upper $(A / B)$ and lower case $(a / b))$. The haploid parasites had also two loci with two alleles and a specific allele combination would allow for an infection of a host with a susceptible genotype. Parasite transmission was based on the frequency of meeting an uninfected susceptible host individual. After infection, selection was calculated per haplotype of the host according to Crow and Kimura (2009) exploring the parameter space for parasite virulence. We excluded dominance effects to simplify population wide estimates of allele selection strengths. Since host individuals can be susceptible to more than one parasite strain we included a transmission process allowing for multiple infections.

To test for the importance of host genome recombination in host-parasite evolution, we included a modifier locus into the model. The modifier locus has two alleles ( $m$ and $M$ ), initially the population is compose of the first allele $(m)$ with the second allele being introduced. We set the initial genome-wide recombination rate to zero (allele $m$ has no effect on recombination), and increase the size of the effect of the second allele $(M)$ on recombination rates (from 0 to 0.5 ). The only difference between these two host-parasite systems was the ploidy of the host male genotype.

\section{Methods}

The model used by (Engelstädter and Bonhoeffer 2009) was redesigned for use with two host systems (haplodiploid and diploid) using the matching allele model (MAM, Wilfert et al 2007b). The two interaction loci are referred to as $\mathbf{A}$ and $\mathbf{B}$, while $\mathbf{M}$ refers to a modifier locus; the alleles were as follows $A / a, B / b$ and $M / m$. Genetic associations between the loci were calculated in the order of $\mathbf{A} \rightarrow \mathbf{B} \rightarrow \mathbf{M}$. The recombination modifier altered the rate of recombination among all loci, such that recombination rates between A-B and B-M were equal. This two loci two allele interaction model, two allele modifier locus and asexual parasite population model differed to that of Engelstädter and Bonhoeffer (2009) by the addition of a parasite transmission model.

Parasite transmission occurs during the first two steps of every iteration of the model (counted as host generations). The first step, upon initialisation, was infection of the host population. This was estimated as the frequency of the parasite in the parent population, multiplied by the proportion of susceptible hosts in the offspring (figure 1, step 1). During the intergenerational infection of host populations it was assumed that the parasite did not distinguish between host genotypes, and would attempt to infect all host genotypes randomly. Hence depending on the frequency of resistant host genotypes, a portion of parasite strains failed to infect a host upon each host generation and were removed from the parasite population. Subsequent calculations 
within the host generation involved probabilities conditional on the proportion of hosts susceptible to the parasite strain (figure 1, step 2). After initial transmission, the within-generation parasite transmission was conducted (figure 1, step 2), after which selection upon the host was calculated (figure 1, step 3). Finally sex and recombination was modelled within the host population, generating the next generation genotype frequencies to be infected by the previously calculated parasite frequencies.

The parasite transmission model required two different processes for within and between generation transmission (Figure 1). Within host generations, transmission was calculated as parasite population growth within the susceptible proportion of the host. Between host generations, parasites were transmitted when they infected a susceptible host, with the previous infected proportions used as the probability of contact between host and parasite. Within each host generation the parasite growth rate $(T)$ is calculated as the degree to which uninfected $\rightarrow$ infected transitions are expected. Therefore, applying the growth rate to the proportion of infected susceptible hosts $\left(p\left(P_{r}\right)\right)$, gives the new proportion of infected individuals. Transmission to the next generation then requires finding the frequency of susceptible host genotypes in the population (the sum of each host genotype frequency $\left.\operatorname{Freq}\left(H_{i}\right)\right)$, which is multiplied by the proportion infected $\left(T \times p\left(P_{r}\right)\right)$.

$$
p\left(P_{r}^{t+1}\right) \leftarrow T \times p\left(P_{r}^{t}\right) \times\left(\sum_{i=1}^{i=n} \operatorname{Freq}\left(H_{i}^{t}\right)\right)
$$

Fitness of the host genotypes was calculated according to the MAM and the proportion of the genotype infected by each parasite strain. In calculating selection coefficients it was assumed that having some mis-matching alleles conferred a selective benefit for the host genotype. In diploid genotypes this was incorporated by subtracting $\frac{\text { virulence }}{4}$ from virulence, while in haploid genotypes $\frac{\text { virulence }}{2}$ was subtracted for each mis-matching allele. Incorporating these selection coefficients with multiple infections required matrices identifying all infection combinations: each matrix had $n$ columns and $2^{n}$ rows, with the elements over the rows and columns being either one of:

\section{proportion surviving infection by parasite strainc $\leftarrow p\left(P_{c}\right) \times\left(1-s_{c}\right)$ proportion of host uninfected by parasite strainc $\leftarrow 1-p\left(P_{c}\right)$} $c$ indicating the column ( $n$ indicating the number of virulent parasite strains). These matrices and the algorithms used to generate the combinations for these matrices are provided in the Online Resources ( 1 and 2, a series of infection matrices and pseudocode of the core algorithms). The probabilities for surviving each infection combination $\left(\prod_{r=n}^{r=1} p\left(s_{r} p\left(P_{r}\right)\right)\right.$, weighted by infection probabilities $\left.p\left(P_{r}\right)\right)$ were then summed to provide the genotypic fitness $\left(1-s_{H}\right)$. 


$$
\left(1-s_{H}\right) \leftarrow \sum_{c=1}^{N}\left(\prod_{r=1}^{n} p\left(s_{r}\left(p\left(P_{r}\right)\right)\right)\right.
$$

Initialisation of the model was performed over a range of settings in three parameters, depending on the question. The three factors were: $i$ ) A factor influencing host mortality (parasite virulence, $s_{r}$ from equation 3 ) over a range of 0 to 1 , weakly to highly virulent parasite strains. ii) The effect of the recombination modifier, varying from no effect (complete linkage) to free recombination ( 0.0 to 0.5 ). iii) The degree of perturbance to the system (away from equilibria); this was done by increasing the skew of the initial parasite frequency distribution.

The results are shown using R (2.13.1, Figure $2 \&$ 5) (R Devel. Core 2011) and gnuplot (4.4, Figures 3 \& 4) (Williams \& Kelley 2010), Figure 1 was produced using Dia (v0.97).

\section{Results}

Individual simulations

Figure 2 compares the RQH frequency oscillations in the diploid host wih those in haplodiploids. Whereas the predicted Red Queen oscillations can be seen in the diploid hosts in a vast range of the parameter space these are completely lacking in the haplodiploids. To better understand of this phenomenon we included extreme parameter values of the virulence, parasite range and recombination modifier.

Analyses of parameter space.

Heatmaps illustrate the parameter space that facilitates the spread of the recombination modifier allele as predicted from the RQ hypothesis (Figure 3): That an arms race between parasites and their hosts will tend to select for elevated recombination rates in the host species. There are clear and pronounced qualitative differences between haplodiploid and diploid hosts regarding the frequency of the recombination modifier. As expected there is a broad parameter space favouring the spread of recombination in the diploid hosts (Figure 3, panel A). This parameter space is much smaller in the haplodiploid host population (panels B \& D, figure 3). Only under both an exceptionally high host mortality and a strong skew in the initial parasite frequency distribution will recombination between the two loci be favoured. The recombination modifier generally evolved to high frequencies when parasite virulence $\left(\mathrm{s}_{R}\right)$ was greater than $50 \%$. The only exception was when parasite virulence was very high $(>0.95)$. This effect was driven by the extinction of the parasite 
Standard model
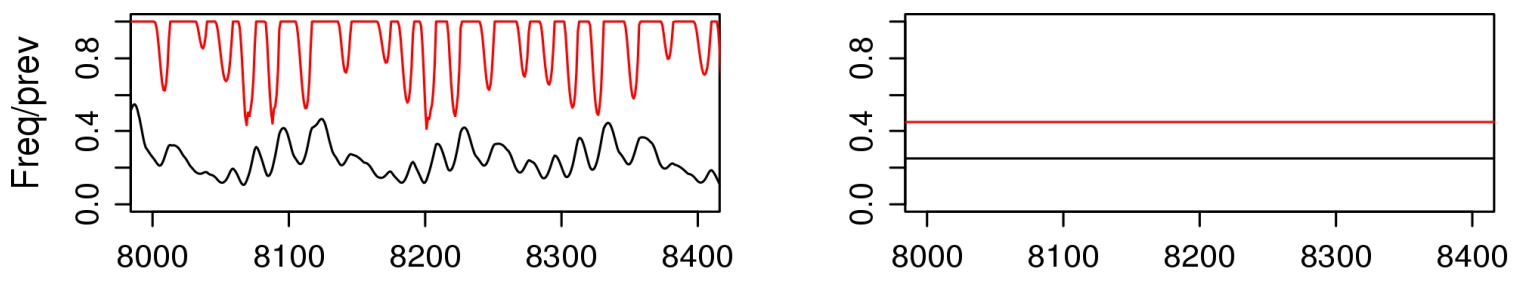

Virulence $=0.8$
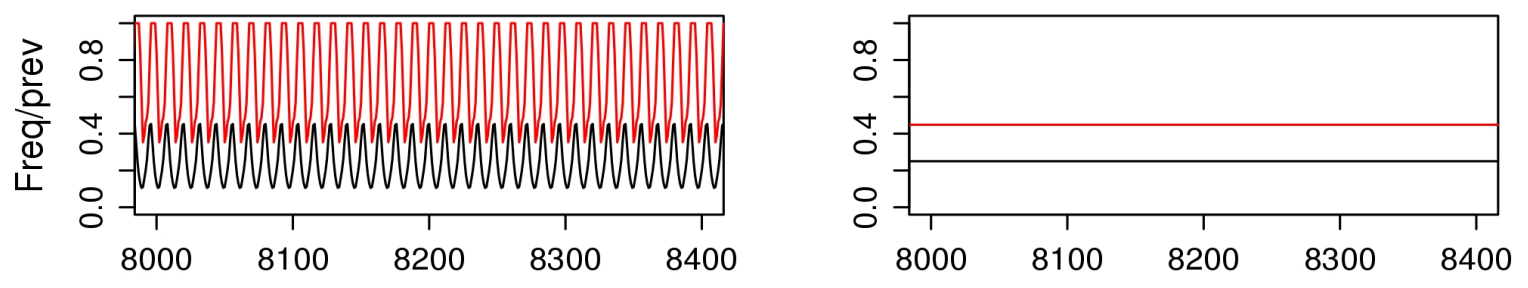

Stronger skew in parasite frequency distribution
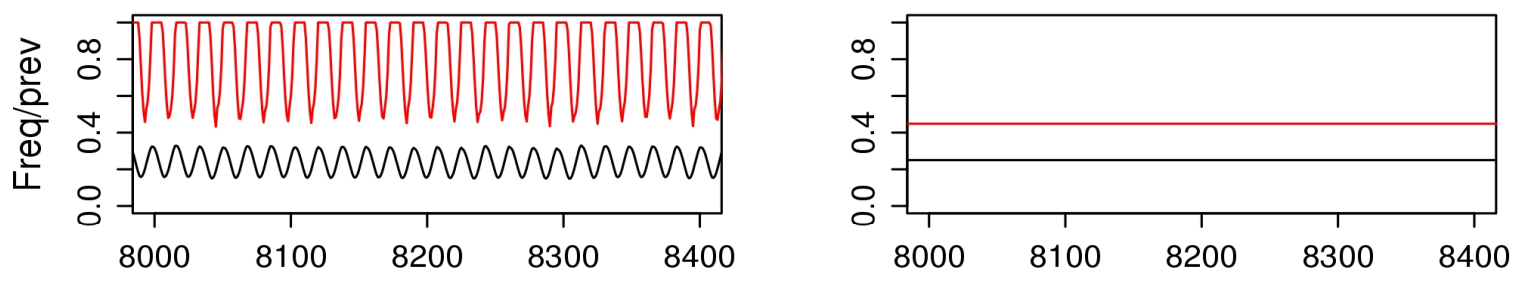

Recombination modifier effect $=0.2$ \& stronger skew in freq. dist.
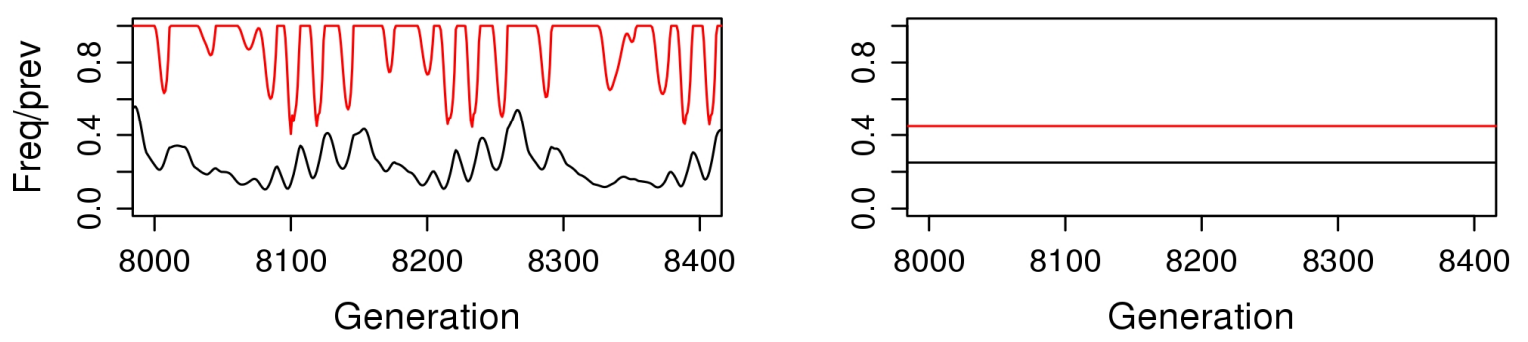

\section{- Host}

2: Plots of the haplotype frequencies from diploid and haplodiploid simulations, and their respective parasite haplotype (only 400 generations shown at steady state for clarity of presentation, 8000 to 8400 from 100, 000 generations). Conditions in the standard run were as follows: virulence $=0.5$, recombination modifier $=0.5$, skewness of parasite frequencies (3.6E-03). In the subsequent figures one or more parameters were changed (the stronger skew is upto 3E-02), these are shown above the corresponding figures. While oscillations in frequency were consistently observed in diploid host populations (over 100, 000 generations), no such dynamics were observed with haplodiploid hosts. In the last graph for the diploid host population, it took upwards of 7000 generations until predictable oscillations occurred. Reduction of the modifier effect was done through the modifier allele $M$ (as allele $m$ has no effect), in the last two graphs $M=0.2$. 
A

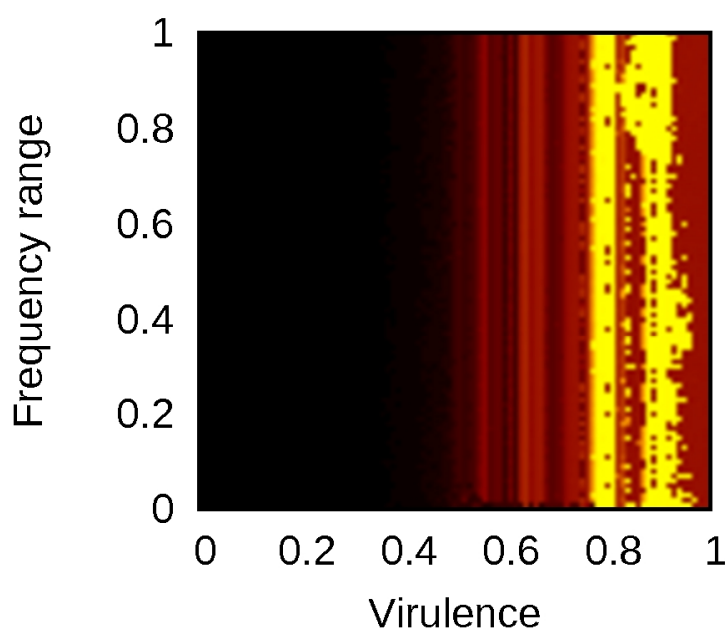

C

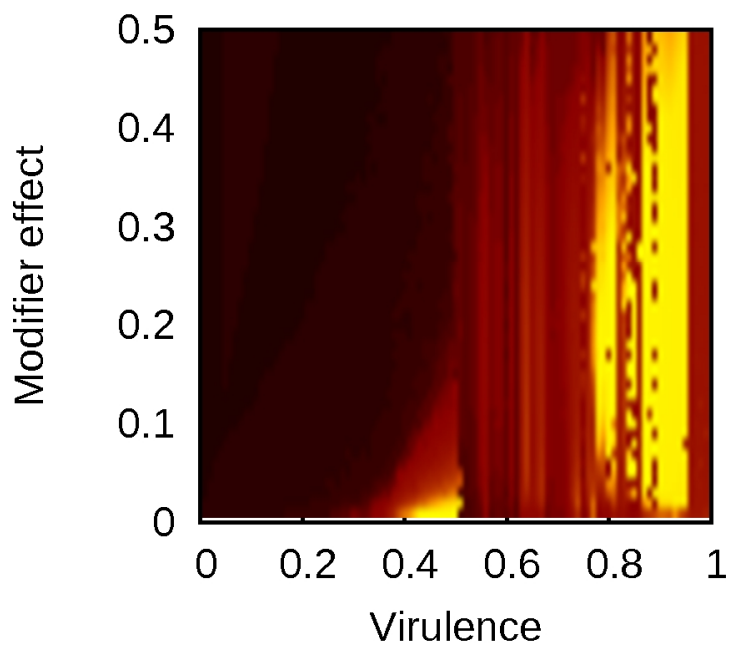

B
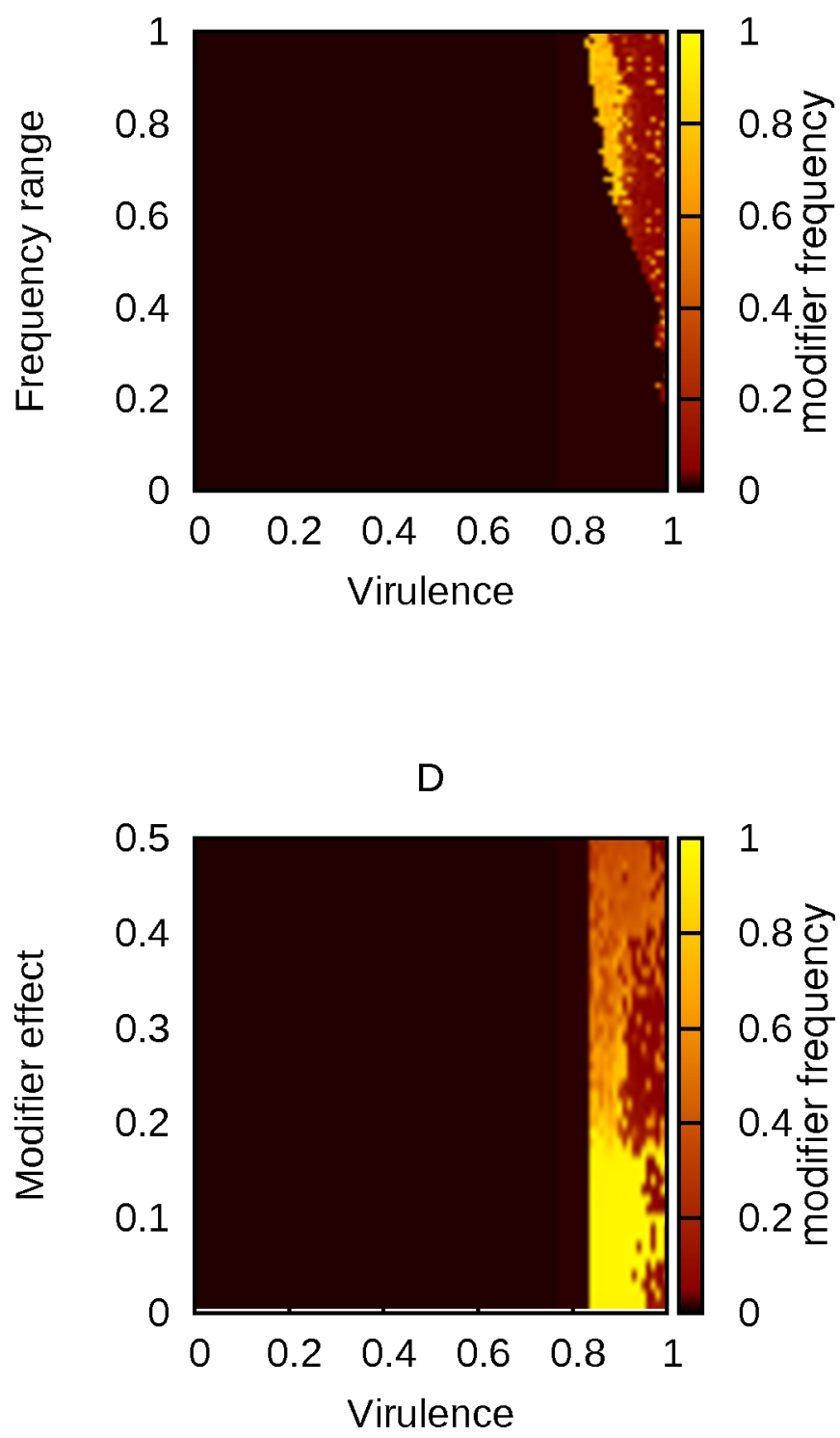

Figure 3: Illustration of recombination modifier frequencies (z/colour-axis) in diploid (graphs in the left column; $A, C$ ) and haplodiploid (graphs in the right column; $B, D$ ) host populations. With rates compared over various levels of parasite virulence ( $x$-axis), different initial parasite frequency distributions $(A \& B)$ and sizes of the recombination modifier effect $(C \& D)$. In graphs $A \& B$ the recombination rate was fixed at 0.2 , while the range of parasite frequencies was fixed to 0.8 in graphs $C \& D$. Over most of parameter space only virulence can be seen to have an effect on the frequency of the recombination modifier (graphs $A \& D$ ). However with a high initial range of parasite frequencies, an affect from the modifier can be seen in both diploid and haplodiploid populations. In diploid host populations lowering the modifier effect increases the range of parameter space in which higher modifier frequencies evolve; while in haplodiploids decreasing the effect size increases the frequency (over a limited parameter space) to which the modifier evolves.

strains early in the simulation, preventing further evolution of the recombination modifier. Selection in the haploids eliminated the matching parasite strains in vast regions of the parameter space.

A role of the size of the effect of the recombination modifier allele (graphs C \& D) an impact was only observed in diploid host populations in conjunction with low parasite virulence $\left(s_{R}\right.$ 

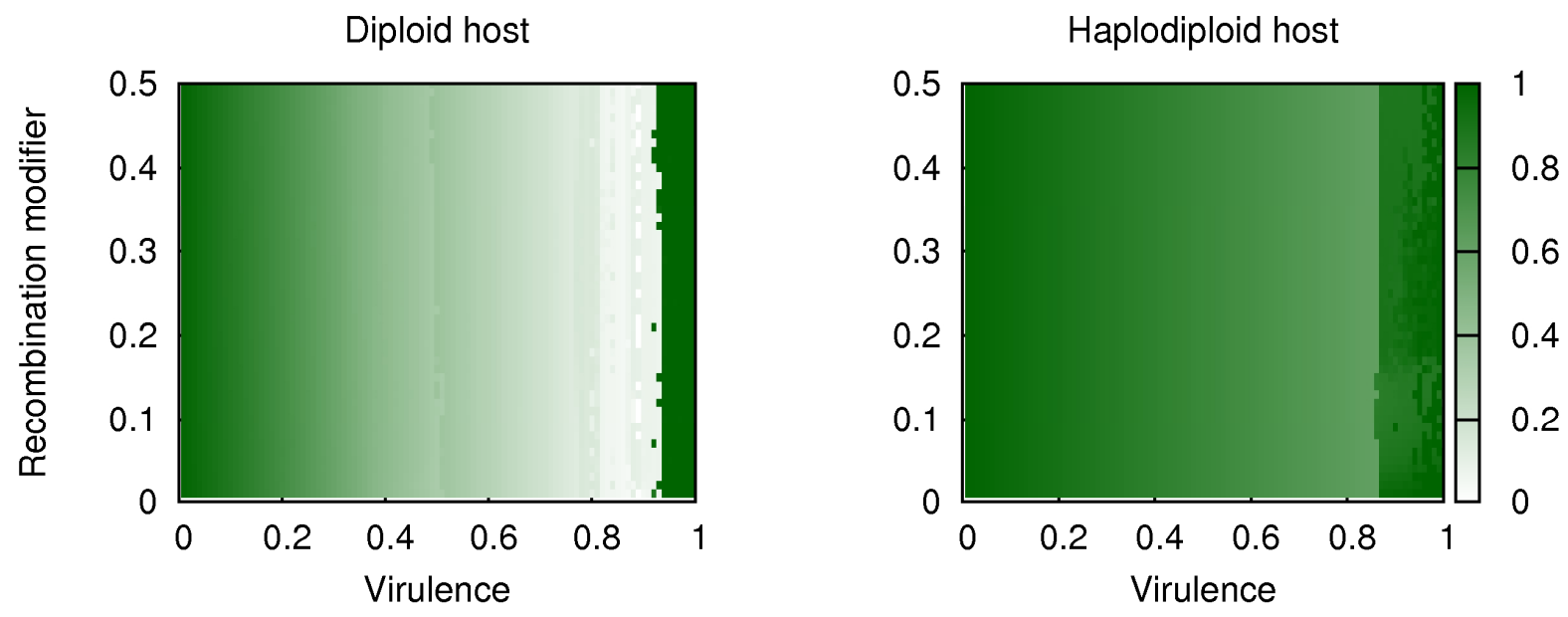

Figure 4: Comparison of fitness over different recombination and virulence schemes, same to that of figure 3 graphs $C \& D$. The size of the recombination modifier effect has little impact on host fitness.

\section{Haplodiploid advantage}

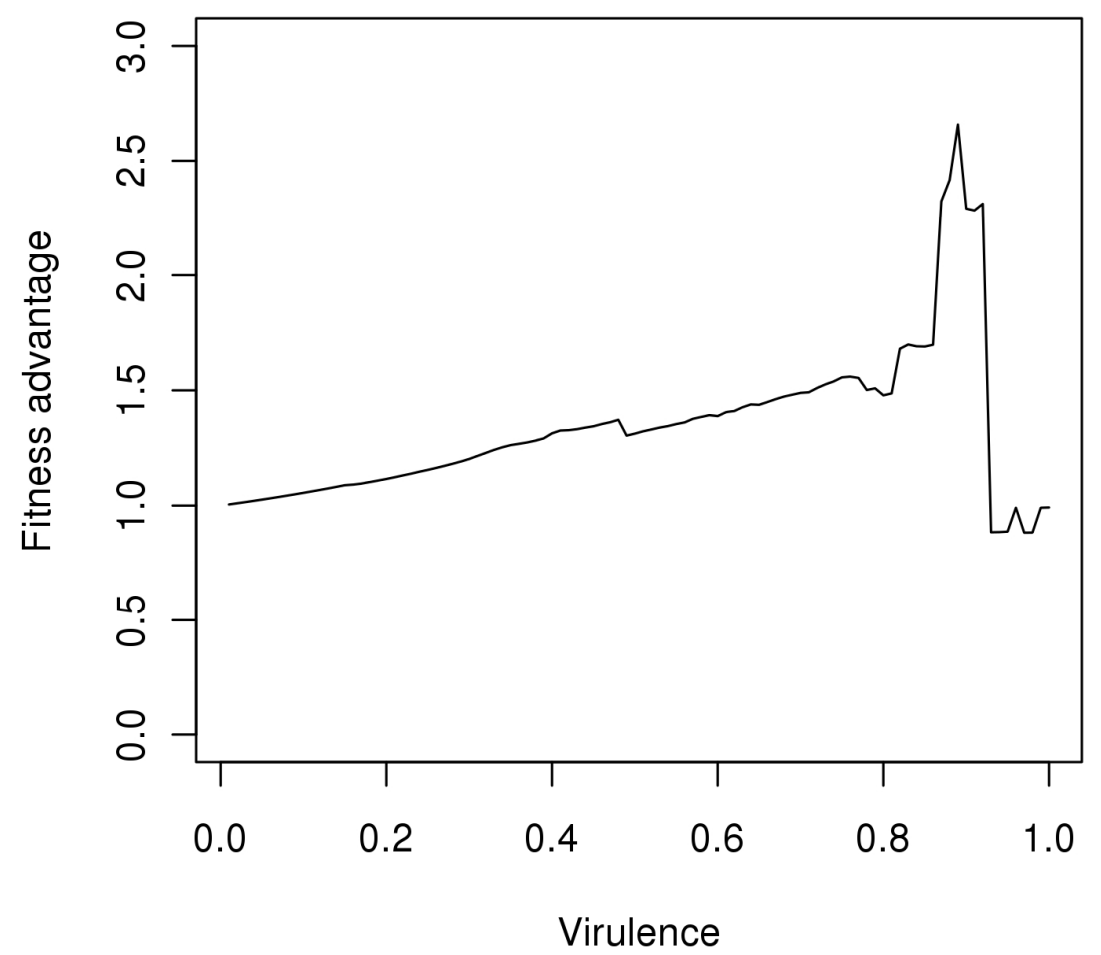

Figure 5: The change in the $x$-fold fitness advantage of haplodiploid lineages against diploids, over virulence. The drop in advantage at high virulence levels occurred from extinction of parasite strains, after successive parasite outbreaks. Only limited conditions show an advantage to diploid host populations.

$<0.5)$. Low recombination modifier effects led to an increase of the Evolutionary Stable Strategy (ESS) frequency of the recombination modifier (panel C). In male-haploid populations the size of 
the recombination modifier effect has no impact on the range of parameter space where recombination is favoured. Surprisingly, with an increasing effect of the recombination modifier ist frequency is reduced in the host population (panel D).

Fitness analysis

We explored the impact of virulence on host fitness by calculating the geometric mean fitness over the size of the recombination modifier effect and parasite virulence (Figure 4 graphs $\mathrm{c}$ and d). These results show a substantial decrease in host fitness over parasite virulence. There were no effects from the size of the recombination modifier effect in neither diploids, nor haplodiploids. The fitness of diploid host populations decreases much faster with increasing parasite virulence than in haplodiploids (graph C, of figure 4). Under high levels of parasite virulence the haplodiploids can have an up to 2.6 fold advantage over diploid hosts (Figure 5).

\section{Discussion}

Individual simulations

We observed marked differences in the dynamics between the diploid and male-haploid host populations in response to infection by multiple parasite strains. The most striking result is the lack of the spread of a recombination modifier and the lack of Red Queen oscillations in haplodiploid host populations. Previous studies analyzed either invasion scenarios (M'Gonigle and Otto 2011), advantages of the host to resistance (Oswald and Nuismer 2007), or conditions favouring parasitism (Nuismer and Otto 2004). The host systems of Mgonigle \& Otto and Nuismer \& Otto were either haploid, diploid (M'Gonigle and Otto 2011); or diploid, tetraploid (Nuismer and Otto 2004). In summary, these papers on population ploidy structure (M'Gonigle and Otto 2011; Nuismer and Otto 2004; Oswald and Nuismer 2007) make the same general findings: $i$ ) haploidy (in the parasite) is almost a universally preferred state for parasitism; ii) in the MAM lower ploidy levels are preferred in the host species; iii) the opposite is true for the IMAM, here higher ploidy levels tend to be preferred. In contrast, our data indicates that oscillations occur in neither the parasite nor host genotype frequencies in male-haploid host populations.

The two model systems presented here only differ in the genomic composition between the two host populations. Hence, any difference in the results must arise from some factor generated by the particular genomic composition. As the results are largely independent of the effect of the recombination modifier (figure $3, \mathrm{D}$ ), the impact of recombination within a single gender can be 
rejected as the cause for an absence of RQ dynamics in the haplodiploid host. Remaining factors are differences in host fitness (between haploid and diploid hosts), and differences in the susceptible host frequencies. Given that host fitness depends on the proportion of the host that is infected, and that parasite transmission depends on susceptible frequencies (Online Resource 3, an algebraic analysis of infection dynamics), it must be the increased variance in susceptible frequencies (resulting from the haploid sex) that drove the differences between the two host populations.

Analyses of parameter space.

The results regarding the recombination modifier in the diploid population were similar to those previously obtained by Agrawal (2009). Independent of the size of the modifier effect, higher recombination rates were generally favoured when virulence was high. In addition however, there is a small range in the virulence parameter space in the diploid host population $\left(0.45 \geq \mathrm{s}_{R} \geq 0.5\right)$ where the size of the effect of the recombination modifier could be small $(0.001<$ effect $<0.02)$ but nevertheless go to fixation.. This does not occur in haplodiploids. Here the establishment of the modifier in the population is much less dependent on its effect size and also the initial parasite frequencies had little effect upon generating oscillations (figure 3, graph A). Parasite virulence was the only driving factor, selecting for the spread of the recombination modifier.

The absence of both parasite and host genotype frequency oscillations in haplodiploids obviously removed recombination as an important driver of selection. This extended over a large region of parameter space and only extremely high virulence $\left(\mathrm{s}_{R} \geq 0.85\right)$ and highly disturbed situations (a large variance in initial parasite frequencies) generated selection for recombination in haplodiploids (figure 3, graph B). Overall the smaller proportion of susceptible haploid male hosts limited the spread of parasite strains, reducing the strength of selection on the host. Further, whenever oscillations were sustained, the relationship between evolutionary stable strategy (ESS) recombination modifier frequencies and recombination modifier effects were negative (figure 3 , graph D). This suggests that sex (or a high recombination rate) is not advantageous per se for haplodiploids in the context of host parasite evolution. Although our results by no means question the role of sexual recombination for selection in host - pathogen systems in dipliod species, they indicate that Red Queen processes are less relevant for maintaining recombination (and sex) in haplodiploids.

Interestingly, extremely high recombination rates have been found in genomes of several eusocial haplodiploid Hymenoptera (Solignac et al. 2004; Meznar et al. 2010; Stolle et al. 2011) which is not predicted in our model. However, living in colonies dramatically changes the population structures for both hosts and parasites fundamentally violating many Hardy Weinberg 
assumptions. For example there will be a dramatic increase of associative contacts among individuals with high genetic similarity. Moreover, selection at the colony level becomes important as does the reduced effective reproductive population sizes in the host. Selection on parasites will rely on both transmission within and between colonies, processes not addressed in our model. All these issues this will clearly need to be considered when explaining the high recombination rates in eusocial insects (Wilfert et al. 2007b; Stolle et al. 2011; Meznar et al 2010; Solignac et al 2004). These factors will also be important for any theory on parasite prevalence (van-Baalen and Beekman 2006; Hughes and Boomsma 2006) and transmission (Walker and Hughes 2009; Otterstatter and Thomson 2007) in and across social haplodiploid species. In addition, it will be essential understand the interactions between genetic diversity and sociality on parasite transmission (Hughes and Boomsma 2006; Walker and Hughes 2009; Baer and Schmid-Hempel 1999; Ugelvig et al. 2010). For now, the robust empirical data on the impact of sociality on parasites outside of human diseases is lacking (Craft and Caillaud 2011).

Our results may have far reaching consequences not only for male-haploid but also for diploid systems with hemizygous sex-chromosomes. Traditionally, a haploid genome (or hemizygous state) has been treated as a condition where individual alleles are under greater selective pressure (Smith 2000). However, our results suggest that under the RQH, the proportion of susceptible individuals in the population is the dominant factor. This may explain the relative absence of selection observed on resistance loci on the X-chromosome (Hill-Burns and Clark 2009), despite evidence for stronger selection over the entire X-chromosome compared to autosomes (Singh et al. 2008). Sex appears to be disadvantageous within male-haploid populations particularly if highly virulent pathogens are present. These results suggest that the high rates of recombination observed in the Hymenoptera may require alternative explanations than that provided by the RQH.

\section{Conclusion}

We observed a marked difference in the host-parasite MAM dynamics between male-haploid and diploid host populations. These differences in host population dynamics have a strong impact on fitness, and the evolution of recombination modifiers. This results in fundamental differences in the predictions of the RQH regarding male-haploid host populations to parasitism, compared to the traditionally studied diploid host populations. Our results suggest that the evolution of high recombination in social Hymenoptera may require an explanation alternative to the RQH.

Acknowledgements We would like to thank the Deutsche Forschungsgemeinschaft (DFG) for funding provided as part of the SPP project number 1399. We would also like to thank Fouks B., 
Murray T. and numerous reviewers for checking the manuscript.

\section{References}

Agrawal A.F. (2009) Differences between selection on sex versus recombination in red queen models with diploid hosts. Evolution 63(8), 2131-2141

Agrawal A.F. \& Otto S.P. (2006) Host-Parasite Coevolution and Selection on Sex through the Effects of segregation. The American Naturalist 168(5), 617-629

Baer B. \& Schmid-Hempel P. (1999) Experimental variation in polyandry affects parasite loads and fitness in a bumble-bee. Nature 397, 151-154

Barton N.H. (1995) A general model for the evolution of recombination. Genetical Research 65, 123-144

Charlesworth B. (1980). The cost of sex in relation to mating system. Journal of Theoretical Biology 84(4), 655-671

Craft M.E. \& Caillaud D. (2011) Network Models: An Underutilized Tool in Wildlife Epidemiology?. Interdisciplinary Perspectives on Infectious Diseases 2011, 1-12

Crow J.F. \& Kimura M. Selection. In Crow \& Kimura (2009). An Introduction to Population Genetics Theory (pp. 173-254) Caldwell, NJ: The Blackburn Press.

Engelstdter J. \& Bonhoeffer S. (2009) Red Queen Dynamics with Non-Standard Fitness Interactions. PLOS computational biology 5, e1000469

Hamilton W.D. (1964). The genetical evolution of social behaviour. I. Journal of Theoretical Biology 7, 1-16

Hill-Burns E.M. \& Clark A.G. (2009) X-Linked Variation in Immune Response in Drosophila melanogaster. Genetics 183, 1477-1491

Hughes W.O.H. \& Boomsma J.J. (2006) Does genetic diversity hinder parasite evolution in social 
insect colonies. Journal of Evolutionary Biology 19, 132-143

Keen N.T. (1990). Gene-for-gene complementarity in plant-pathogen interactions. Annu. Rev. Genet. 24, 447-463

Meznar, E.R., Gadau J., Koeniger N. \& Rueppell O. (2010) Comparative Linkage Mapping Suggests a High Recombination Rate in All Honeybees. Journal of Heredity 101: s118-126

M'Gonigle L.K. \& Otto S.P. (2011) Ploidy and the evolution of parasitism. Proc. R. Soc. B 278, $2814-2822$

Niehuis O., Gibson J.D., Rosenberg M.S., Pannebakker B.A., Koevoets T., Judson A.K., Desjardins C.A., Kennedy K., Duggan D., Beukeboom L.W., van de zande L., Shuker D.M., Werren J.H. \& Gadau J. (2010) Recombination and its Impact on the genome of the Haplodiploid Parasitoid Wasp Nasonia. PLOS One 5(1), e8597

Normark B.B. (2003) The Evolution of Alternative Genetic Systems in Insects. Annual Review of Entomology. 48, 397-423

Nuismer S.L. \& Otto S.P. (2004) Host-parasite interactions and the evolution of ploidy PNAS 101, $11036-11039$

Oswald B.P. \& Nuismer S.L. (2007) Neopolyploidy and pathogen resistance. Proc. R. Soc. B. 274, 2393-2397

Otterstatter M.C. \& Thomson J.D. (2007) Contact networks and transmission of an intestinal pathogen in Bumble bee (Bombus impatiens) colonies. Oecologia 154, 411-421

Otto S.P. \& Nuismer S.L. (2004) Species Interactions and the Evolution of Sex. Science 304, 1018

Peters A.D. \& Lively C.M. (1999) The Red Queen and fluctuating epistasis: a population genetic analysis of antagonistic coevolution. American Naturalist 154, 393-405

Peters A.D. \& Lively C.M. (2007) Short- and long-term benefits and detriments to recombination under antagonistic coevolution. Journal of Evolutionary Biology 20, 1206-1217 
R Development Core Team. R: A Language and Environment for Statistical Computing. R Foundation for Statistical Computing. http://www.R-project.org Vienna, Austria (2011) ISBN 3900051-07-0.

Salathé M., Kouyos R.D., Regoes R.R. \& Bonhoeffer S. (2008) Rapid parasite adaptation drives selection for high recombination rates. Evolution 62(2), 295-300

Schmid-Hempel P. \& Jokela J. (2002) Socially Structured Populations and Evolution of Recombination under Antagonistic Coevolution. The American Naturalist 160(3), 403-408

Singh N.D., Larracuente A.M. \& Clark A.G. (2008) Contrasting the Efficacy of Selection on the X and Autosomes in Drosophila. Molecular Biology and Evolution 25(2), 454-467

Smith N.G.C. (2000) The evolution of haplodiploidy under inbreeding. Heredity 84, 186-192

Solignac M., Vautrin D., Baudry E., Mougel F., Loiseau A. \& Cornuet J.M. (2004) A MicrosatelliteBased Linkage Map of the Honeybee, Apis mellifera L. Genetics 167, 253-262

Stolle E., Wilfert L., Schmid-Hempel R., Schmid-Hempel P., Kube M., Reinhardt R. \& Moritz R.F.A. (2011) A second generation genetic map of the bumblebee Bombus terrestris (Linnaeus, 1758) reveals slow genome and chromosome evolution in the Apidae. BMC Genomics 12, 48

Sutton J.T., Nakagawa S., Robertson B.C. \& Jamieson I.G. (2011) Disentangling the roles of natural selection and genetic drift in shaping variation at MHC immunity genes. Molecular Ecology 20, $4408-4420$

Takahashi Y., Morita S., Yoshimura J. \& Watanabe M. (2011) A geographic cline induced by negative frequency-dependent selection. BMC Evolutionary Biology 11, 256-266

Ugelvig L.V, Kronauer D.J.C., Schrempf A., Heinze J. \& Cremer S. (2010) Rapid anti-pathogen response in ant societies relies on high genetic diversity. Proceedings of the Royal Society B 277, $2821-2828$

van-Baalen M. \& Beekman M. (2006) The Costs and Benefits of Genetic Heterogeneity in 
Resistance against Parasites in Social Insects. The American Naturalist 167(4), 568-577

Walker T.N. \& Hughes W.O.H. (2009) Adaptive social immunity in leafcutting ants. Biology Letters $5,446-448$

Wilfert L., Gadau J. \& Schmid-Hempel P. (2007a) The genetic architecture of immune defense and reproduction in male Bombus terrestris Bumblebees. Evolution 61(4), 804-815

Wilfert L., Gadau J. \& Schmid-Hempel P. (2007b) Variation in genomic recombination rates among animal taxa and the case of social insects. Heredity 98, 189-197

Williams T. \& Kelley C. (2010) GNUPLOT 4.4: An Interactive Plotting Program.

Http://gnuplot.sourceforge.net/ 